\section{Acknowledgments}

We would like to thank the Consultants concerned for allowing us to study their cases and for helpful criticism. We are grateful to Dr G. A. K. Missen for his helpful assistance with the pathology, also to Mr Fitzpatrick and the Photographic Department, Guy's Hospital, for help in the preparation of the figures and Miss Rosemary Edmonds for typing the manuscript.

\section{References}

Adams, W.E. \& Bloch, R.G. (1949) Hemangioma of the mediastinum, report of a case. Archives of Surgery, 48, 126.

Attar, S. \& Cowley, R.A. (1964) Hemangioma of the mediastinum: collection, review and case report. American Surgeon, 30, 141.

Baltaxe. H.A. \& Constable, W.C. (1968) Mediastinal lymph node visualisation in the absence of intrathoracic disease. Radiology, 90, 94.

Blades, B. (1946) Mediastinal tumours. Report of cases treated at the Army Thoracic Surgery Centers in United States. Annals of Surgery, 123, 749.

Bцоом, H.J.G. (1964) In: Tumours of the Kidney and Ureter, Monographs on Neoplastic Disease Series (Ed. by D. W. Smithers), p. 316. E. S. Livingstone: London.

BoyD, D.P. \& Midell, A.I. (1968) Mediastinal cysts and tumors. An analysis of 96 cases. Surgical Clinics of North America, 48, 493.

Bewer, L.A. \& Dolley, F.S. (1949) Tumours of the mediastinum. American Review of Tuberculosis, 60, 419.

Carlens, E. (1965) Mediastinoscopy. Annals of Otology, Rhinology and Laryngology, 74, 1102.

Ellis, F.H. Kirklin, J.W. \& WoOlner. L.B. (1955) Hemangioma of the mediastinum. Journal of Thoracic Surgery, 30, 181 .
Everson, R.C. \& Cole, W.H. (1959) Spontaneous regression of malignant disease. Journal of the American Medical Association, 142, 1758.

GreenWell, F.P. \& Wright, F.W. (1965) Rotational tomography. Clinical Radiology, 16, 377.

Heuer, G.J. \& ANDRUS, W.DEW. (1940) Surgery of mediastinal tumors. American Journal of Surgery, 50, 146.

Martin, L.W. (1964) Angiomas in infants and children. American Journal of Surgery, 107, 115.

NOHL-OSER, H.C. (1972) An investigation of the anatomy of the lymphatic drainage of the lungs. Annals of the Royal College of Surgeons of England, 51, 157.

Peace, P.K. \& Price, J.L. (1973) Preoperative tomographic assessment of the mediastinum in bronchial carcinoma. Thorax, 28, 367.

Pearson, F.G. \& Kergin, F.G. (1965) Mediastinoscopy: a method of biopsy in the superior mediastinum. Journal of Thoracic and Cardiovascular Surgery, 49, 11.

Riches, E.W., Griffiths, I.H. \& ThackRAY, A.C. (1951) New growths of kidney and ureter (B.A.U.S. series). British Journal of Urology, 23, 297.

Riches, E.W. (1964) In: Tumours of the Kidney and Ureter, Monographs on Neoplastic Disease (Ed. by D. W. Smithers), p. 367. E. S. Livingstone: London.

RuSBY, N.L. (1944) Dermoid cysts and teratomata of the mediastinum. Journal of Thoracic Surgery, 13, 169.

Smithers, D.W. (1962) Spontaneous regression of tumours. Clinical Radiology, 13, 132.

Tandon, P.L., Kumar, M. \& Haffiz, M.A. (1963) Metastases from renal cell carcinoma twenty years after nephrectomy. A case report. British Journal of Urology, 35, 30.

Wychulis, A.R., Payne, W.S., Clagett, O.T. \& Woolner, L.B. (1971) Surgical treatment of mediastinal tumours. 40 year experience. Journal of Thoracic and Cardiovascular Surgery, 62, 379.

Postgraduate Medical Journal (February 1975) 51, 96-99.

\title{
Neonatal listeriosis
}

\author{
S. VARIEND \\ M.B., M.R.C.P., D.C.H.
}

\section{BLUMENTHAL M.B., Ch.B.}

\section{The Jessop Hospital for Women, Sheffield}

\section{Summary}

Three cases of neonatal listeriosis are reported. This local incidence in a busy neonatal unit over a 10-year period would agree with other reports that the condition is rare in the United Kingdom. Two cases had septicaemia and one developed septicaemia and meningitis, contrasting with the previous view that the commonest presentation is with meningitis, and could be due to the greater use of blood cultures in babies suspected of having infection. The mode of delivery differed in each case which allowed certain conclusions to be made regarding pathogenesis of the condition. Thus evidence is presented against fetal infection through an ascending route or while passing through

Correspondence: Dr S. Variend, City Hospital, Nottingham NG5 1PB. an infected birth canal. Maternal bacteraemia with a transplacental spread of infection would seem more likely. The possible use of Gentamicin in the treatment of this condition is discussed and the better prognosis with septicaemia, compared with meningitis, is emphasized.

\section{Introduction}

Listeria monocytogenes is a pleomorphic, motile, Gram-positive rod which shows a characteristic tumbling movement at $25^{\circ} \mathrm{C}$, growth at low temperature, $\beta$-haemolysis on blood agar and an ability to ferment certain sugars. There are four recognized serotypes, of which types 1 and 4 are most commonly encountered in Britain (Public Health Laboratory Service, 1969). 
L. monocytogenes is frequently confused with other bacteria and it has been suggested that in many laboratories it is incorrectly identified and discarded as a contaminant (Louria, 1970). Furthermore, the organism is known to decolorize easily (Barber and Okubadejo, 1965) and has often been mistaken for Gram-negative bacteria (Insley and Hussain, 1964; Selinger and Becker, 1955). It has been suggested that the rising incidence in America may be due to improved methods of identification (Driscoll, Gorbach and Feldman, 1962).

According to the figures of the Public Health Laboratory Service forty cases under 1 year of age were reported from the United Kingdom between 1967 and 1972 (Public Health Laboratory Service, 1972 , personal communication). While this incidence is higher than the number of published cases would lead one to believe, it is still a reminder that the condition is much less common in Britain than in the United States of America (Louria, 1970) or Germany (Gray, Seeliger and Potel, 1963).

\section{Case 1}

The mother was a 35 -year-old gravida 2 , with a normal child born after caesarean section for cephalopelvic disproportion. The present pregnancy was uncomplicated and ended in an elective caesarean section at term. This produced a male infant (birth weight $2475 \mathrm{~g}$ ), with an Apgar score of 5 at $1 \mathrm{~min}$. During the early post-partum period the mother developed symptoms suggestive of a urinary tract infection, but investigations were inconclusive. Blood culture on the mother was not performed. On the fifth day the infant developed a vesicular eruption but remained well until the fourteenth day when he was found to be pale, with a vacant stare. Treatment was started with penicillin and streptomycin after lumbar puncture and blood culture had been performed. A thorough search for organisms on a Gram smear of CSF was unsuccessful but $L$. monocytogenes was cultured from both the blood and CSF. The organism was sensitive to penicillin, sulphamethazine, streptomycin and chloramphenicol. Because of delayed clinical improvement treatment was changed to chloramphenicol and sulphamethazine, and, in addition, chloramphenicol and hydrocortisone were given intrathecally. Treatment was continued for 12 days and he was discharged after a month. When seen at the age of 2 months hydrocephalus was diagnosed and a Spitz-Holter valve was introduced. At the age of 13 years, apart from a degree of mental subnormality, he was found to be progressing well.

\section{Case 2}

A 21-year-old primigravida had an uncomplicated twin pregnancy which ended in spontaneous labour at 35 weeks. Twin 1 , a female infant weighing $1701 \mathrm{~g}$ was born as a vertex presentation, with an Apgar score of 10 at $1 \mathrm{~min}$. Twin 2 was born after internal podalic version and breech extraction. This resulted in a female infant (birth weight, $1701 \mathrm{~g}$ ) with an Apgar score of 7 at $1 \mathrm{~min}$. The fetal membranes were dichorionic. After $24 \mathrm{hr}$ twin 2 developed a series of cyanotic attacks and was investigated for possible infection. Treatment was started with ampicillin and cloxacillin. An organism with the properties of $L$. monocytogenes was isolated on blood culture but not from the CSF which was normal. The blood count and chest X-ray were also normal. The organism was sensitive to penicillin $(0.5 \mathrm{u} / \mathrm{ml})$ and ampicillin $(0.8 \mu \mathrm{g} / \mathrm{ml})$. In view of rapid clinical improvement, the initial choice of antibiotics was continued for 10 days. A full investigation was carried out on twin 1 and the mother but did not reveal infection. When seen at the age of 4 years both children were well and developing normally.

\section{Case 3}

A 41-year-old gravida 4, with three previous normal pregnancies had an uneventful pregnancy which ended at 36 weeks. Delivery was normal and produced a male infant (birth weight, $2780 \mathrm{~g}$ ), with an Apgar score of 8 at $1 \mathrm{~min}$. There was respiratory distress with cyanosis at $24 \mathrm{hr}$. The liver edge was 2.5 finger breadths below the right costal margin and the spleen tip was palpable. The chest X-ray and CSF were normal. Treatment was started with gentamicin and penicillin. The blood culture grew $L$. monocytogenes. The organism was sensitive to penicillin $(0.5$ $\mathrm{u} / \mathrm{ml})$ and gentamicin $(0.03 \mu \mathrm{g} / \mathrm{ml})$. The response was dramatic and treatment was continued for 10 days. A vaginal swab from the mother on the seventh day post partum also yielded $L$. monocytogenes, and she was treated with a 10-day course of oral penicillin. When seen after 3 months both mother and baby were well.

\section{Discussion}

There can be little doubt that infants acquire the infection from the mother who may or may not develop symptoms during the last months of pregnancy. Maternal symptoms are non-specific and blood cultures taken at this time may be positive for Listeria (Hood, 1961). Studies have shown involvement of the placenta in some cases of perinatal listeriosis (Scott and Henderson, 1968), suggesting that a transplacental route of infection was involved. Evidence that other routes of infection may be involved is shown in a prospective study of twenty-one affected infants in which only five cases showed infection of the placenta (Alison and Sarrut, 1968).

Autopsy findings in infants have demonstrated widespread pneumonic and gastro-intestinal lesions 
suggesting inhalation and swallowing of infected material (Becroft et al., 1971). In such cases it is suggested that the organism invades the amniotic fluid from a contiguous site such as the cervix. It is also possible that the amniotic fluid may be contaminated by infected fetal urine following fetal septicaemia (Gray et al., 1963). In addition, infants are said to be infected while passing through the birth canal, and the organism is commonly isolated from the vagina and cervix in affected cases. Since case 1 was born by elective caesarean section it is evident that this route was not responsible. In a study of twin placentae Benirschke (1960) found no case in which only twin 2 or its placenta showed evidence of infection, whereas there was such evidence for twin 1 in seventeen of his cases. Because of the proximity of the fetal membranes of the first twin to the cervical os he concluded that the most likely route of such infection was an ascending one. This is at variance with our case of twin pregnancy in which twin 2 was found to have evidence of infection; twin 1 was unaffected. Loda and Sell (1963) also described a case of Listeria septicaemia in the second of binovular twins at 7 days; twin 1 was normal.

Several reports have shown the organism to be sensitive to many anti-microbial agents. Many of these agents are toxic to the newborn and individual strains may be resistant to penicillin (Edmunds, Nicholson and Douglas, 1957; McKinnon and Swithinbank, 1961). Evidence on ampicillin suggests that it may be a useful therapeutic agent (Macnair, White and Graham, 1968).

On the other hand, reports on gentamicin are scanty. In vitro studies have shown more effective killing of the Listeria organism with gentamicin and ampicillin together, than with either drug alone (Gordon, Barret and Clark, 1972). Stock cultures from our first two cases were recently tested and shown to be sensitive to gentamicin at $0.03 \mu \mathrm{g} / \mathrm{ml}$. It is difficult to say to what extent this antibiotic was responsible for the prompt recovery of case 3 and only further experience with gentamicin will evaluate its true role in treating this condition. Because of its poor penetration into the cerebrospinal fluid it cannot ordinarily be recommended for the treatment of listerial meningitis, but may be useful if given intrathecally.

Antibiotic treatment is often necessary in suspected neonatal infections before sensitivity reports are available. The in vitro sensitivity reports on gentamicin and the successful outcome of case 3 should give reasonable assurance that a combination of gentamicin and penicillin will provide adequate cover against Listeria infection.

Symptoms in the newborn are non-specific as illustrated in our cases. It is said that Listeria infection in the newborn most commonly presents with meningitis (Barber and Okubadejo, 1965), but cases with septicaemia are being more commonly described. Less common stigmata include rhinitis, conjunctivitis and skin lesions which may be papular, vesicular, pustular or, rarely, purpuric. It could well be that the skin eruption on day 5 in case 1 represented the first clinical evidence of infection but unfortunately investigations were not carried out at that stage.

Our cases were diagnosed over a period when there were 36,812 deliveries, including 905 stillbirths. Survival in all our cases contrasts with the mortality usually reported in this condition, $50 \%$ (Barrie, Snodgrass and Coleman, 1967) and $54 \%$ (Becroft et al., 1971). However, some of these reports have included a higher incidence of infants with very low birth weight, a common outcome of affected pregnancies. There have been few reports on follow-up of patients with neonatal listeriosis. Our cases with septicaemia alone survived without sequelae, whereas case 1 developed hydrocephalus, a common complication of Listeria meningitis in the newborn (Gray and Killinger, 1966). In a recent review of twenty-nine cases of neonatal listeriosis including five with meningitis, eight were found to have evidence of damage to the central nervous system (Degen and Goldenbaum, 1965); of these there were three cases of hydrocephalus.

\section{Acknowledgments}

We wish to thank Dr J. A. Black for permission to publish cases and for having read and criticized the paper. Thanks are also due to $\mathrm{Dr}$ J. Colquhoun and $\mathrm{Mr} \mathrm{K}$. Megson for their advice and reports on bacteriological investigations.

\section{References}

Alison, F. \& SARrut, S. (1968) Neonatal listeriosis: increased frequency or greater awareness? Clinical Pediatrics, 7, 309.

Barber, M. \& Okubadejo, O.A. (1965) Maternal and neonatal listeriosis: report of case and brief review of literature of listeriosis in man. British Medical Journal, ii, 735.

Barrie, H., Snodgrass, G.J.A.I. \& Coleman, J.C. (1967) Neonatal Listeria monocytogenes meningitis and septicaemia. Proceedings of the Royal Society of Medicine, 60, 671.

Becroft, D.M.O., Farmer, K., Seddon, R.J., Sowden, R., StewarT, J.H., Vines, A. \& Wattie, D.A. (1971) Epidemic listeriosis in the newborn. British Medical Journal, iii, 747.

BeNiRSCHKe, K. (1960) Routes and types of infection in the fetus and newborn. American Journal of Diseases in Childhood, 99, 714.

Degen, R. \& Goldenbaum, C. (1965) A follow-up of 29 cases of listeriosis of the newborn. Deutsche Medizinische Wochenschrift, 90, 1898.

Driscoll, G.S., Gorbach, A. \& Feldman, D. (1962) Congenital listeriosis: Diagnosis from placental studies. Obstetrics and Gynecology, New York, 20, 216.

Edmunds, P.N., Nicholson, D.N. \& Douglas, D.M. (1957) Two cases of listerial meningitis in infants. British Medical Journal, ii, 188. 
Gordon, R.C., Barret, F.F. \& Clark, D.J. (1972) Influence of several antibiotics, singly and in combination, on the growth of Listeria monocytogenes. Journal of Pediatrics, 80, 667.

Gray, M.L. \& Killinger, A.H. (1966) Listeria monocytogenes and listeric infections. Bacteriological Reviews, 30, 309.

Gray, M.L., Seeliger, H.P.R. \& Potel, J. (1963) Perinatal infections due to Listeria monocytogenes: do these affect subsequent pregnancies? Clinical Pediatrics, 2, 614.

Hood, M. (1961) Listeriosis as an infection of pregnancy manifested in the newborn. Pediatrics, 27, 390.

INSLEY, J. \& HuSSAIN, Z. (1964) Listerial meningitis in infancy. Archives of Disease in Childhood, 39, 278.

LODA, F.L. \& SEll, S.H.W. (1963) Meningitis in the newborn infants due to Listeria monocytogenes: report of three cases. Southern Medical Journal, 56, 872.
Louria, D.B. (1970) Listeriosis. Internal Medicine Digest, 5 39.

Macnair, D.R., White, J.D. \& Graham, J.M. (1968) Ampicillin in the treatment of Listeria monocytogenes meningitis. Lancet, i, 16.

McKinnon, D. \& Swithinbank, J.M. (1961) Meningitis due to Listeria monocytogenes. Lancet, ii, 516.

Public Health Laboratory Service (1969) Epidemiology. British Medical Journal, ii, 751.

Public Health Laboratory Service (1972) Epidemiology. British Medical Journal, ii, 415.

Selinger, B. \& Becker, P.P. (1955) Listeria meningitis. Pediatrics, 16, 500.

Scott, J.M. \& Henderson, A. (1968) A case of listeriosis of the newborn. Journal of Medical Microbiology, 1, 97.

\title{
Eosinophilic granuloma of the ileum
}

\author{
A. MYERS \\ M.B., M.R.C.P. \\ DAPHNE M. HUMPHREYS \\ M.B., M.R.C.P. \\ R. C. N. Williamson \\ F.R.C.S.
}

\section{Department of Medicine, Royal Berkshire Hospital, Reading}

\begin{abstract}
Summary
A case of eosinophilic granuloma of the ileum is described in association with a high $(50 \%)$ eosinophil count. A review of published suggested classifications, aetiology and therapy is made.
\end{abstract}

\section{Case report}

Mr R.B. aged 61 years was admitted as an emergency to the Royal Berkshire Hospital, Reading, in October 1972. He had been in good health until he returned from holiday in Ibiza 2 months before admission. Since then he had complained of daily frontal headaches, lower abdominal pain unrelated to food, and occasional vomiting. There had been no change in bowel habit although the stools had been lighter than usual. He was anorexic and had lost $12 \mathrm{lb}$ in weight. There was no known contact with infective hepatitis and no injections or administration of drugs. There was a past history of bacillary dysentery in Beirut in 1916.

On examination he was ill, thin and sallow, with early clubbing and slight epigastric tenderness. His temperature was $37.5^{\circ} \mathrm{C}$, pulse $85 / \mathrm{min}$ and blood pressure $100 / 80 \mathrm{mmHg}$.

He was extensively investigated. The only abnormal findings were a marked peripheral eosinophilia

Correspondence: Dr A. Myers, 102 Cavendish Drive, Leytonstone, London E11.
(7000 eosinophils $/ \mathrm{mm}^{3}$ ) and a weakly positive (1 : 32) fluorescent amoebic antibody test.

The following investigations were normal or negative. Haemoglobin; ESR; prothrombin time; urea and electrolytes; blood sugar; serum calcium, serum proteins; alkaline phosphatase; LDH, SGOT; bilirubin and urine bile salts; serum immunoglobulins; Paul Bunnell; LE cells; Australia antigen; antibody titres for leptospirosis, brucellosis, salmonellosis, toxoplasmosis, psittacosis and a wide variety of viruses, Mycoplasma pneumoniae and Rickettsia burnetii; urine and stool culture. No ova or cysts were seen in the stools and occult bloods were negative. A liver biopsy was normal except for trapped eosinophils in the liver sinuses. Liver and pancreatic scans and ECG were also normal.

He was treated with consecutive 5-day courses of metoclopramide $10 \mathrm{mg}$ t.d.s. and metronidazole 400 $\mathrm{mg}$ t.d.s., and then paromomycin 500,000 u q.d.s., with marked improvement in his symptoms. Following discharge he gained weight, still suffered from occasional lower abdominal pains and was slightly constipated, but continued to improve. The eosinophilia persisted (Fig. 1). All other biochemical tests were normal.

He was readmitted on 26 January to the Surgical Department, Royal Berkshire Hospital, with a 24-hr history of severe constant pain in the right iliac fossa 